\title{
Substitution mapping of the major quantitative trait loci controlling stigma exsertion rate from Oryza glumaepatula
}

\section{Quanya Tan}

South China Agricultural University

\section{Tuo Zou}

South China Agricultural University

Mingmin Zheng

South China Agricultural University

Yuerong Ni

South China Agricultural University

Xin Luan

South China Agricultural University

Xiaohui Li

South China Agricultural University

\section{Weifeng Yang}

South China Agricultural University

\section{Zifeng Yang}

South China Agricultural University

Haitao Zhu

South China Agricultural University

\section{Ruizhen Zeng}

South China Agricultural University

\section{Guifu Liu}

South China Agricultural University

\section{Shaokui Wang}

South China Agricultural University

\section{Xuelin Fu}

South China Agricultural University

Guiquan Zhang ( $\boldsymbol{\nabla}$ gqzhang@scau.edu.cn )

https://orcid.org/0000-0003-3322-2753 
Keywords: stigma exsertion, outcrossing, quantitative trait locus, single-segment substitution line, wild species, rice

Posted Date: April 9th, 2020

DOI: https://doi.org/10.21203/rs.3.rs-21523/v1

License: (c) (1) This work is licensed under a Creative Commons Attribution 4.0 International License. Read Full License

Version of Record: A version of this preprint was published at Rice on June 9th, 2020. See the published version at https://doi.org/10.1186/s12284-020-00397-1. 


\section{Abstract}

Background: Stigma exsertion rate (SER) is a key determinant for outcrossing ability of male sterility lines (MSLs) in hybrid rice seed production. Outcrossing ability in cultivated rice varieties has diminished during the process of domestication, while wild Oryza species keep strong outcrossing ability. Here, we detected the quantitative trait loci (QTLs) controlling SER using a set of single-segment substitution lines (SSSLs) derived from 0. glumaepatula, a wild Oryza species.

Results: Seven QTLs for SER, qSER-1a, qSER-1b, qSER-3a, qSER-3b, qSER-5, qSER-9 and qSER-10, were located on 5 chromosomes. qSER-1a and qSER-1b were located on chromosome 1. qSER-3a and qSER-3b were mapped on chromosome 3 , and $q S E R-3 b$ was further located at an interval of $931.0 \mathrm{~kb}$ by secondary substitution mapping. qSER-5, qSER-9 and $q S E R-10$ were identified on chromosomes 5, 9 and 10, respectively, and $q S E R-9$ was delimited to a region of $608.2 \mathrm{~kb}$ by secondary substitution mapping. The additive effects of the 7 QTLs ranged from $10.6 \%$ to $14.8 \%$, and the additive contribution variances explained by each of the QTLs were from $36.3 \%$ to $50.6 \%$, which were higher than those of most loci for SER reported previously.

Conclusions: qSER-1a and qSER-1b were novel loci for SER on chromosome 1. All of the 7 QTLs had major effects on SER. The major QTLs of SER will help to develop MSLs with strong outcrossing ability.

\section{Background}

As one of the most important cereal crops, rice plays a key role in sustaining world food security and supplying more than $20 \%$ calories intake as a staple (Zuo and Li 2014). The success of hybrid rice commercialization results largely in improving yield of rice (Guo et al. 2017). However, cultivated rice is predominantly self-fertilizing with less than 1\% natural cross-pollination (Virmani and Athwal 1973). Outcrossing ability in cultivated rice varieties has diminished along with changes in the morphology of rice flowers during the process of domestication. Previous studies have established that exserted stigma, as a main mating organ, could keep viable for several days after flowering (Long and Shu 2000; Zhou et al. 2017a), and then get more opportunities to catch pollens (Rahman et al. 2017; Zhang et al. 2018). Therefore, stigma exsertion rate (SER) is a key determinant for outcrossing ability of male sterility lines (MSLs) in hybrid rice seed production.

Wild Oryza species have strong outcrossing ability. It is reported that perennial wild rice had higher outcrossing than annual types (Oka and Morishima 1967). Cultivated rice tends to have a shorter stigma than the annual wild species. The annual wild species have shorter stigma than their perennial progenitors. The wider variability for stigma, style, and their total lengths in wild species of rice might have developed simultaneously during evolution and domestication (Marathi et al. 2015). In the past two decades, many of QTLs controlling SER and related traits have been located on 12 chromosomes of rice genome from various genetic resources (Marathi and Jena 2015; Zhou et al. 2017a; Liu et al. 2019). However, only 2 wild Oryza species were used to identify QTLs of SER until now, and O. rufipogon was 
most commonly used. Xiong et al. (1999) reported an QTL for extruding stigma on chromosome 6 using an $\mathrm{F}_{2}$ population derived from a cross between $O$. sativa, Aijiao Nante, and $O$. rufipogon, P16. Li et al. (2001) found two QTLs of SER on chromosomes 5 and 8 by a backcross- $F_{1}$ population between $O$. rufipogon and Guichao 2, an elite indica rice. Uga et al. (2003a) identified two QTLs for rate of exserted stigma between the indica line Pei-kuh and W1944 of 0 . rufipogon. Huang et al. (2012) found 8 QTLs for SER using the $271 \mathrm{~F}_{2}$ lines from the cross between $O$. sativa, Guangluai-4, and 0 . rufipogon. Bakti and Tanaka (2019) detected five QTLs for SER using an $\mathrm{F}_{2}$ population generated from a cross between the japonica rice cultivar 'Akidawara' and 'W0120' of $O$. rufipogon. From another wild species, $O$. longistaminata, several QTLs of SER were identified from an introgression line of the wild species in the genetic background of Asian cultivated rice (Li et al. 2010).

O. glumaepatula is one of the AA-genome wild Oryza species indigenous to Central and South America (Doi et al. 2008). Recently, Stein et al. (2018) elucidated that $O$. glumaepatula is the sister group only of African 0 . barthii and 0 . glaberrima based on the genome sequence alignment. Several hybrid pollen sterility genes between $O$. sativa and $O$. glumaepatula have been identified, such as S12, S22, S23, S27 and S28 (Sano 1994; Sobrizal et al. 2000a, b; Sobrizal and Yoshimura 2001, 2002; Sakata et al. 2014; Fang et al. 2019). It was found that the endosperm of $O$. glumaepatula had the high levels of total protein, albumin, and glutelin protein fractions and amino acids, showing the potential to increase the nutritional quality of rice storage protein (Santos et al. 2013). O. glumaepatula also showed the rapid internodal elongation potential under partial submergence (Sasayama et al. 2018), as well as its important yield and yield component traits (Zhang et al. 2015; Bhatia et al. 2017). It is important to note that $O$. glumaepatula had a longer stigma length, which is favorable for outcrossing (Marathi et al. 2015).

In order to detect QTLs for traits of agronomic importance in O. glumaepatula, 168 SSSLs were developed by using 0 . glumaepatula as donors and Huajingxian74 (HJX74), an indica variety of 0 . sativa as recipient. The total length of the substituted segments in the set of SSSLs was $3636.4 \mathrm{cM}$, which were distributed on 12 chromosomes (Zhao et al. 2019). In the present study, we used the set of SSSLs to identify QTLs for SER. Seven of the QTLs were located on 5 chromosomes. The QTLs had higher additive effects. These major QTLs of SER from O. glumaepatula will be helpful for developing of MSLs with strong outcrossing ability.

\section{Results}

SER in the SSSLs derived from 0. glumaepatula

At the first, a set of 168 SSSLs derived from 0 . glumaepatula were investigated for SER and 9 of the SSSLs with higher SER were selected. The 9 SSSLs were then tested their SER for 5 cropping seasons. Compared with the HJX74 recipient, the 9 SSSLs were showed higher SER at the $P \leq 0.001$ level in every cropping season. Average SER of the 9 SSSLs in the 5 cropping seasons were from $48.1-60.8 \%$ with 18.5-31.2\% higher than that of the control HJX74 (Fig. 1 and Additional file: Table S1). 
The chromosomal substituted segments from 0. glumaepatula in the 9 SSSLs were detected by molecular markers. The substituted segments in the 9 SSSLs were detected on chromosomes 1, 3, 5, 9 and 10, respectively. The estimated lengths of substituted segments were from $1467.0 \mathrm{~kb}$ to $16410.5 \mathrm{~kb}$ (Additional file: Table S2 and Table S3).

Eight of agronomic traits in the 9 SSSLs were investigated in two cropping seasons. Compared with HJX74, most of the traits in the SSSLs had no significant difference, except the plant height in SG25, the heading date in SG153, and so on (Additional file: Table S4).

\section{Two QTLs for SER were mapped on chromosome 1}

Four SSSLs with high SER, SG18, SG22, SG23 and SG25, carried substituted segments on chromosome 1. SG18 had a substituted segment at the interval from markers RM140 to RM5853 with the estimated length of $10741.8 \mathrm{~kb}$ (Fig. 1 and Additional file: Table S3). Therefore, the substituted segment of SG18 carried an QTL for SER, named qSER-1a.

Other three SSSLs, SG22, SG23, and SG25 carried substituted segments in the region of 29385.9$41082.0 \mathrm{~kb}$ and the substituted segments were overlapped. SG22 had the shortest substituted segment with the estimated length of $2611.3 \mathrm{~kb}$ from markers RM403 to RM6648. The estimated lengths of substituted segments were $5874.5 \mathrm{~kb}$ in SG23, and $11059.7 \mathrm{~kb}$ in SG25. These results indicated that the three SSSLs carried another QTL for SER, named $q S E R-1 b$, which located in the substituted segment of SG22 with the interval of $2611.3 \mathrm{~kb}$ (Fig. 2 and Additional file: Table S3).

Because the $q S E R-1 b$ was linked to $S d 1, S G 25$ carried the $S d 1$ in the substituted segment from $O$. glumaepatula and showed tall in plant height with $160.3 \mathrm{~cm}$ in FCS and $135.7 \mathrm{~cm}$ in SCS. SG22 and SG23 carried shorter substituted segments and had no Sd1 from O. glumaepatula. Although SG22 and SG23 were semi-dwarf in plant height, the SER had no significant difference with SG25 (Fig. 2 and Additional file: Table S4). These results indicated that the $q S E R-1 b$ of SER was not influenced by plant height.

\section{Two QTLs for SER were mapped on chromosome 3}

Two SSSLs with high SER, SG59 and SG65, carried substituted segments on chromosome 3. SG59 had a substituted segment at the interval from markers RM282 to InDel3 with the estimated length of $4632.1 \mathrm{~kb}$ (Fig. 1 and Additional file: Table S3). Therefore, the substituted segment of SG59 carried an QTL for SER, named $q S E R-3 a$.

The SSSL SG65 carried a substituted segment at the interval of 26747.5-35843.1 kb on chromosome 3 . The substituted segment was in a different region from that in SG59. Therefore, SG65 had another QTL for SER, qSER-3b, in its substituted segment (Fig. 3b, Additional file: Table S3).

To narrow the interval of $q S E R-3 b, 5$ secondary SSSLs were developed from an $\mathrm{F}_{2: 3}$ population derived from the cross of HJX74/SG65. The secondary SSSL, SG65-14, carried a substituted segment in the 
region from markers RM15678 to ID03MC07 on the left. Two of the secondary SSSLs, SG65-48 and SG65-61, had substituted segments in the regions from PSM132 and RM186 to RM442 on the right, respectively. The three secondary SSSLs showed lower SER as HJX74. Other two secondary SSSLs, SG65-9 and SG65-55, carrying substituted segments covered the region from markers ID03MC07 to RM186 showed higher SER as SG65. These results indicated that the $q S E R-3 b$ was narrowed to an interval of $931.0 \mathrm{~kb}$ between markers ID03MC07 and RM186 (Fig. 3).

\section{Secondary substitution mapping of qSER-9}

One SSSL, SG135, carried a substituted segment on chromosome 9. Therefore, the substituted segment had an QTL for SER, qSER-9. To further map $q S E R-9,5$ secondary SSSLs were developed from an $\mathrm{F}_{2: 3}$ population from the cross of HJX74/SG135. Two of the secondary SSSLs, SG135-26 and SG135-30, carried respectively substituted segments in the regions from ID09M06 to ID09M16 and RM105 on the left. The secondary SSSL, SG135-6, had a substituted segment in the region from ID09M23 to RM3600 on the right. The three secondary SSSLs showed lower SER as HJX74. Other two secondary SSSLs, SG135-44 and SG135-68, carried substituted segments from ID09M16 to ID09M23, and had higher SER as SG135. These results indicated that the $q S E R-9$ was delimited to an interval of $608.2 \mathrm{~kb}$ between ID09M16 and ID09M23 (Fig. 4).

\section{Other QTLs for SER identified in the SSSLS}

One SSSL, SG85, carried a substituted segment from markers RM7444 to RM291 on chromosome 5 with the estimated length of $16410.5 \mathrm{~kb}$. Therefore, the SSSL had an QTL for SER, $q S E R-5$, in the substituted segment (Fig. 1 and Additional file: Table S3).

Another SSSL, SG153, carried a substituted segment from markers RM484 to RM25886 on chromosome 10 with the estimated length of $1467.0 \mathrm{~kb}$ and had an QTL for SER, qSER-10, in the substituted segment (Fig. 1 and Additional file: Table S3).

Summarily, a total of 7 QTLs for SER was mapped on 5 chromosomes. Among of them, chromosomes 1 and 3 each carried two of the QTLs, and chromosomes 5, 9 and 10 each carried one of the QTLs (Fig. 5).

\section{The additive effects of QTLs for SER identified in the SSSLS}

The additive effects of the 7 QTLs for SER, qSER-1a, qSER-1b, qSER-3a, qSER-3b, qSER-5, qSER-9 and qSER-10, ranged from $10.6-14.8 \%$ and the additive contribution variances explained by each of the QTLS for SER were from 36.3-50.6\%. Among of them, $q S E R-10$ had the highest additive effect of $14.8 \%$ and the highest additive contribution variance of $50.6 \%$ (Table 1 ). 
Table 1

Additive effects of QTLs for stigma exsertion rate detected in the SSSLS

\begin{tabular}{|c|c|c|c|c|c|c|}
\hline QTL & Chr. & Interval (kb) & Estimated length (kb) & $P$ value & $A(\%)$ & ACV (\%) \\
\hline qSER-1a & 1 & 12291.6-23033.4 & 10741.8 & $2.5 \mathrm{E}-08$ & $11.9 \pm 0.8$ & $40.7 \pm 3.7$ \\
\hline$q S E R-1 b$ & 1 & 29608.2-32219.6 & 2611.3 & $3.6 \mathrm{E}-06$ & $12.4 \pm 1.8$ & $42.5 \pm 6.3$ \\
\hline qSER-3a & 3 & $11656.8-16288.9$ & 4632.1 & $1.2 \mathrm{E}-07$ & $11.1 \pm 1.1$ & $38.0 \pm 4.4$ \\
\hline$q S E R-3 b$ & 3 & 27677.1-28608.1 & 931.0 & $2.4 \mathrm{E}-07$ & $10.7 \pm 1.2$ & $37.1 \pm 5.5$ \\
\hline qSER-5 & 5 & $3216.5-19626.9$ & 16410.5 & 1.3E-09 & $13.5 \pm 1.6$ & $46.5 \pm 6.6$ \\
\hline qSER-9 & 9 & $14342.2-14950.4$ & 608.2 & 3.1E-07 & $10.6 \pm 1.0$ & $36.3 \pm 4.2$ \\
\hline qSER-10 & 10 & 21132.3-22599.3 & 1467.0 & $1.2 \mathrm{E}-10$ & $14.8 \pm 2.0$ & $50.6 \pm 7.2$ \\
\hline
\end{tabular}

\section{Discussion}

In the past two decades, many of QTLs that affect stigma exsertion were identified in rice genome. The QTLs were distributed across all 12 chromosomes (Marathi and Jena 2015; Zhou et al. 2017a; Liu et al. 2019). Among of them, a limited number of QTLs for SER were identified from the wild Oryza species, $O$. rufipogon (Xiong et al. 1999; Li et al. 2001; Uga et al. 2003a; Huang et al. 2012; Bakti and Tanaka 2019) and O. longistaminata (Li et al. 2010). In the present study, we detected 7 QTLs of SER on 5 chromosomes using a set of SSSLs derived from O. glumaepatula. On chromosome 1, qSER-1a and qSER-1b are novel loci, in which region no other QTLs for SER were reported previously. On chromosome 3, qSER-3a was located in the QTL cluster of qES3 (Miyata et al. 2007), PES-3 (Yue et al. 2009), qSPE3 (Feng et al. 2010) and qSSE3 ( $\mathrm{Li}$ et al. 2014a). qSER-3b was mapped in the region locating of $q T S E-3 b$ (Li et al. 2014b) and qSER-3.2 (Xu et al. 2019). On chromosome 5, the region of $q S E R-5$ covered those of qPEST-5 (Li et al. 2001), qPES-5 (Deng et al. 2010), qTSE-5a (Li et al. 2014b), TSE (Zhou et al. 2017a) and qSER-5.1 (Xu et al. 2019). On chromosome 9, the $q S E R-9$ region was partly overlapped with the segment of $q P E S-9$ from T821B, an introgression line from O. longistaminata (Li et al. 2010). In addition, the qSER10 interval on chromosome 10 was overlapped with the region of qDSE-10 (Li et al. 2014a). Although most of the loci identified in this study were located in the same regions as the previously reported loci, it was noted that the additive effects of the loci identified from 0 . glumaepatula were generally greater. The additive effects of the seven loci identified in this study were between $10.6 \%$ and $14.8 \%$, while the additive effects of most of the previously reported loci were less than $8.0 \%$. It indicated that the QTLs from 0 . glumaepatula had major effects on SER, which will be favorable for molecular breeding of MSLs with greater ability for outcrossing. 
During the process of domestication, cultivated rice has already lost some traits of natural outcrossing by varying from a vegetative to sexual manner of reproduction (Parmar et al. 1979). Wild Oryza species have a strong outcrossing ability due to their larger stigma, longer style, greater exsertion of the stigma, and longer periods of spikelet opening (Marathi et al. 2015; Marathi and Jena 2015). Previous studies revealed dominant differences for SER and floral traits between cultivated rice and wild rice (Virmani and Athwal 1973; Uga et al. 2003b). It was found that O. glumaepatula had longer of stigma length than cultivated rice (Marathi et al. 2015). In the present study, we identified 7 QTLs for SER with major effects from the SSSLs derived from 0 . glumaepatula. It indicated that the favorable alleles for outcrossing traits of larger stigma, longer style, greater exsertion of the stigma, and longer periods of spikelet opening, and so on, which were lost in cultivated rice, could be mined in wild Oryza species.

In the past two decades, we have constructed an SSSL library by the using of HJX74 as the recipient and 43 accessions from 7 species, including 5 wild Oryza species of AA genome as donors (Zhang et al. 2004; Xi et al. 2006; Zhang 2019). The SSSLs have been used to map QTLs (Zhang et al. 2012; Yang et al. 2016; Zhou et al. 2017b), to clone genes (Wang et al. 2012; Sui et al. 2019), to analyze allelic variation (Teng et al. 2012; Cai et al. 2013) and to conduct molecular breeding by design (Dai et al. 2015, 2016; Luan et al. 2019). In the present study, the SSSLs derived from O. g/umaepatula were used to identified the QTLs for SER. Seven QTLs of SER with major effects were located on 5 chromosomes. These results indicated that the HJX74-SSSL library is a powerful tool for mapping of QTLs of agronomic importance and identifying of naturally occurring alleles in germplasm, particularly for mining of the favorable alleles lost in cultivated rice from wild species.

\section{Conclusion}

Using the SSSLs derived from O. glumaepatula, seven major QTLs controlling SER, qSER-1a, qSER-1b, qSER-3a, qSER-3b, qSER-5, qSER-9 and $q S E R-10$, were located on 5 chromosomes by substitution mapping. qSER-1a and qSER-1b were novel loci for SER, in which regions no other QTLS of SER were reported previously. Compared with the previously reported loci, the 7 QTLs have major additive effects and additive contribution variances. The major QTLs will be favorable for molecular breeding of MSLs with strong outcrossing ability. The favorable alleles for outcrossing traits which were lost in cultivated rice could be mined in the wild Oryza species.

\section{Methods}

\section{Materials and filed experiment}

The set of SSSLs derived from O. glumaepatula (Zhao et al. 2019) and their parents HJX74 and $O$. glumaepatula were grown in the paddy fields at the experimental farm of South China Agricultural University in Guangzhou $\left(23^{\circ} 07^{\prime} \mathrm{N}, 113^{\circ} 15^{\prime} \mathrm{E}\right)$, China. The materials were grown in 5 cropping seasons from 2016 to 2018, two cropping seasons per year, the first cropping season (FCS) from late February to middle July and the second cropping season (SCS) from late July to middle November. Single-seedling 
transplanting was applied in field experiment. Each plot had four rows with ten single plants per row. Standard cultivation practices and controlling of diseases and insect pests were followed the typical methods in South China.

\section{Molecular markers and PCR protocol}

SSR markers labeled "RM" were selected from online resources (https://archive.gramene.org/markers/). The "PSM" and "Indel" markers were designed using software of Primer Premier 5.0 (Lalitha 2000). DNA was extracted from the fresh leaves of each plant referencing a reported method (Murray and Thompson 1980). The PCR products were amplified and analyzed on $6 \%$ denatured polyacrylamide gel, and detected using the silver staining using the methods described by Fang et al. (2019).

\section{Phenotyping of traits and statistical analysis}

The stigma exsertion (SE) was subdivided into single stigma exsertion (SSE) and dual stigma exsertion (DSE). The SER refers to total stigma exsertion rate, including single stigma exsertion rate and dual stigma exsertion rate. For investigating SER, 8-10 main panicles were sampled from plants in 1-2 days after flourishing florescence of florets. The SER per panicle was calculated based on visual aspect of exserted stigmas (Xu et al. 2019). Agronomic traits were measured by using the YTS rice phenotypic facility (Yang et al. 2014). Differences of traits were determined using one-way ANOVA. SPSS 23.0 and Origin 9.0 plus (https://www.originlab.com) were applied to analyze experimental data and draw figures in this paper.

\section{Mapping of QTLs}

The lengths of substituted segments of SSSLs were calculated by the previous method (Xi et al. 2006; He et al. 2017; Zhao et al. 2019). The minimum length $\left(L_{\min }\right)$ of a substituted segment refers to the length between two markers of donor genotype at the end of the substitution segment. The maximum length

$\left(L_{\max }\right)$ refers to the length between two markers of background genotype flanking the substitution segment. The estimated length $\left(L_{\text {est }}\right)=\left(L_{\min }+L_{\text {max }}\right) / 2$. The QTLs for SER were mapped by the substitution mapping method (Eshed and Zamir 1995; Wissuwa et al. 2002). Linkage map of QTLs was drawn by using MapChart2.2 (https://www.wur.nl/en/show/Mapchart.htm). QTLs were named by the method of McCouch et al. (1997).

\section{Abbreviations}

SER: Stigma exsertion rate; QTL: Quantitative trait locus; SSSL: Single-segment substitution line; MAS: Marker-assisted selection; MSL: Male sterility line; FSC: First cropping season; SCS: Second cropping season

\section{Declarations}

Funding 
This work was supported by grants from the National Natural Science Foundation of China $(91435207$ and 91735304), and from the Guangzhou Science and Technology Program Key Projects (201607020040 and 201804020086).

Availability of data and materials

All data generated or analysed during this study are included in this published article and its additional information files.

Authors' contributions

GZ and XF conceived and designed the experiments and supervised the research works. QT performed most of the experiments and prepared the data and the draft of manuscript. TZ, YN, MZ, XL, XL, WY and ZY performed a part of experiments. HZ, RZ, GL, SW and GZ participated in material development. GZ analyzed the data and wrote the manuscript. All authors read and approved the final manuscript.

Ethics approval and consent to participate

Not applicable.

Consent for publication

Not applicable.

Competing interests

The authors declare that they have no competing interests.

\section{References}

1. Bakti C, Tanaka J (2019) Detection of dominant QTLs for stigma exsertion ratio in rice derived from Oryza rufipogon accession 'W0120'. Breed Sci 69:143-150

2. Bhatia D, Joshi S, Das A, Vikal Y, Sahi GK, Neelam K, Kaur K, Singh K (2017) Introgression of yield component traits in rice (Oryza sativa ssp indica) through interspecific hybridization. Crop Sci 57:1557-1573

3. Cai J, Liao QP, Dai ZJ, Zhu HT, Zeng RZ, Zhang ZM, Zhang GQ (2013) Allelic differentiations and effects of the $R f 3$ and $R f 4$ genes on fertility restoration in rice with wild abortive cytoplasmic male sterility. Biol Plant 57:274-280

4. Dai Z, Lu Q, Luan X, Cai J, Zhu H, Liu Z, Zeng R, Zhang Z, Wang S, Zheng L, Li J, Zhang G (2015) Development of a platform for breeding by design of CMS lines based on an SSSL library in rice (Oryza sativa L.). Euphytica 205:63-72

5. Dai Z, Lu Q, Luan X, Ouyang L, Guo J, Liang J, Zhu H, Wang W, Wang S, Zeng R, Liu Z, Zhang Z, Zhu X, Zhang G (2016) Development of a platform for breeding by design of CMS restorer lines based on 
an SSSL library in rice (Oryza sativa L.). Breed Sci 66:768-775

6. Deng YD, Ying JZ, Shi YY, Xiao CL, Zhang HQ (2010) Mapping of QTLs for percentage of exserted stigma in rice. Journal of Hunan Agricultural University (Natural Sciences) 36:373-376 (Chinese with English abstract)

7. Doi K, Yasui H, Yoshimura A (2008) Genetic variation in rice. Curr Opin Plant Biol 11:144-148

8. Eshed Y, Zamir D (1995) An introgression line population of Lycopersicon pennellii in the cultivated tomato enables the identification and fine mapping of yield-associated QTL. Genetics 141:11471162

9. Fang C, Li L, He R, Wang D, Wang M, Hu Q, Ma Q, Qin K, Feng X, Zhang G, Fu X, Liu Z (2019) Identification of $S 23$ causing both interspecific hybrid male sterility and environment-conditioned male sterility in rice. Rice 12:10

10. Feng LL, Jing YH, Huang C, Xu ZJ, Chen WF (2010) QTL analysis of percentage of exserted stigma in rice (Oryza sativa L.). North Rice 40:20-22 (Chinese with English abstract)

11. Guo L, Qiu F, Gandhi H, Kadaru S, De Asis EJ, Zhuang J, Xie F (2017) Genome-wide association study of outcrossing in cytoplasmic male sterile lines of rice. Sci Rep 7:3233

12. He N, Wu R, Pan X, Peng L, Sun K, Zou T, Zhu H, Zeng R, Liu Z, Liu G, Wang S, Zhang G, Fu X (2017) Development and trait evaluation of chromosome single-segment substitution lines of $O$. meridionalis in the background of $O$. sativa. Euphytica 213:281

13. Huang X, Kurata N, Wei X, Wang Z, Wang A, Zhao Q, Zhao Y, Liu K, Lu H, Li W, Guo Y, Lu Y, Zhou C, Fan D, Weng Q, Zhu C, Huang T, Zhang L, Wang Y, Feng L, Furuumi H, Kubo T, Miyabayashi T, Yuan X, Xu Q, Dong G, Zhan Q, Li C, Fujiyama A, Toyoda A, Lu T, Feng Q, Qian Q, Li J, Han B (2012) A map of rice genome variation reveals the origin of cultivated rice. Nature 490:497-501

14. Lalitha S (2000) Primer premier 5. Biotech Software Internet Report 1:270-272

15. Li C, Sun CQ, Mu P, Chen L, Wang XK (2001) QTL analysis of anther length and ratio of stigma exsertion, two key traits of classification for cultivated rice (Oryza sativa L.) and common wild rice (Oryza rufipogon Griff.). Acta Genetica Sinica 28:746-751 (Chinese with English abstract)

16. Li HB, Gao FY, Zeng LH, Li QX, Lu XJ, Li ZH, Ren JS, Su XW, Ren GJ (2010) QTL analysis of rice stigma morphology using an introgression line from Oryza longistaminata L. Molecular Plant Breeding 8:1082-1089 (Chinese with English abstract)

17. Li P, Feng F, Zhang Q, Chao Y, Gao G, He Y (2014a) Genetic mapping and validation of quantitative trait loci for stigma exsertion rate in rice. Mol Breeding 34:2131-2138

18. Li P, Su G, Feng F, Wang P, Yu S, He Y (2014b) Mapping of minor quantitative trait loci (QTLs) conferring fertility restoration of wild abortive cytoplasmic male sterility and QTLs conferring stigma exsertion in rice. Plant Breeding 133:722-727

19. Liu Y, Zhang A, Wang F, Kong D, Li M, Bi J, Zhang F, Wang J, Luo X, Pan Z, Yu X, Liu G, Luo L (2019) Fine mapping a quantitative trait locus, $q S E R-7$, that controls stigma exsertion rate in rice (Oryza sativa L.). Rice 12:46 
20. Long LH, Shu K (2000) Increasing outcrossing rate of indica hybrid rice. Journal of Hunan Agricultural University 2000:167-170 (Chinese with English abstract)

21. Luan X, Dai Z, Yang W, Tan Q, Lu Q, Guo J, Zhu H, Liu G, Wang S, Zhang G (2019) Breeding by design of CMS lines on the platform of SSSL library in rice. Mol Breeding 39:126

22. Marathi B, Jena KK (2015) Floral traits to enhance outcrossing for higher hybrid seed production in rice: present status and future prospects. Euphytica 201:1-14

23. Marathi B, Ramos J, Hechanova SL, Oane RH, Jena KK (2015) SNP genotyping and characterization of pistil traits revealing a distinct phylogenetic relationship among the species of Oryza. Euphytica 201:131-148

24. McCouch SR, Cho YG, Yano M, Paul E, Blinstrub M, Morishima H, Kinosita T (1997) Report on QTL nomenclature. Rice Genetics Newsletters 14:11-12

25. Miyata M, Yamamoto T, Komori T, Nitta N (2007) Marker-assisted selection and evaluation of the QTL for stigma exsertion under japonica rice genetic background. Theor Appl Genet 114:539-548

26. Murray MG, Thompson WF (1980) Rapid isolation of high molecular weight plant DNA. Nucleic Acids Res 8:4321-4325

27. Oka HI, Morishima H (1967) Variations in the breeding systems of wild rice, Oryza perennis. Evolution 21:249-258

28. Parmar KS, Siddiq EA, Swaminathan MS (1979) Variation in components of flowering behavior of rice. Indian Journal of Genetics Plant Breeding 39:542-550

29. Rahman MH, Zhang Y, Sun L, Zhang K, Rahman MS, Wu W, Zhan X, Cao L, Cheng S (2017) Genetic mapping of quantitative trait loci for the stigma exsertion rate in rice (Oryza sativa L.). Journal of Integrative Agriculture 16:1423-1431

30. Sakata M, Yamagata Y, Doi K, Yoshimura A (2014) Two linked genes on rice chromosome 2 for $F_{1}$ pollen sterility in a hybrid between Oryza sativa and O. glumaepatula. Breed Sci 64:309-320

31. Sano Y (1994) Pollen-killers in rice. Breed Sci 44:298

32. Santos KFDN, Silveira RDD, Martin-Didonet CCG, Brondani C (2013) Storage protein profile and amino acid content in wild rice Oryza glumaepatula. Pesquisa Agropecuária Brasileira 48:66-72

33. Sasaki A, Ashikari M, Ueguchi-Tanaka M, Itoh H, Nishimura A, Swapan D, Ishiyama K, Saito T, Kobayashi M, Khush GS, Kitano H, Matsuoka M (2002) Green revolution: a mutant gibberellinsynthesis gene in rice. Nature 416:701-702

34. Sasayama D, Okishio T, Hirano T, Fukayama H, Hatanaka T, Akimoto M, Azuma T (2018) Internodal elongation under submergence in the Amazonian wild rice species Oryza g/umaepatula: The growth response is induced by hypoxia but not by ethylene. Plant Growth Regul 85:123-132

35. Sobrizal MY, Sanchez PL, Ikeda K, Yoshimura A (2000a) Identification of a gene for male gamete abortion in backcross progeny of Oryza sativa and Oryza glumaepatula. Rice Genetics Newsletters 17:59-61 
36. Sobrizal MY, Sanchez PL, Ikeda K, Yoshimura A (2000b) Mapping of $F_{1}$ pollen semi-sterility gene found in backcross progeny of Oryza sativa L. and Oryza glumaepatula Steud.. Rice Genetics Newsletters 17:61-63

37. Sobrizal MY, Yoshimura A (2001) Mapping of a gene for pollen semi-sterility on chromosome 8 of rice. Rice Genetics Newsletters 18:59-61

38. Sobrizal MY, Yoshimura A (2002) Mapping of pollen semi-sterility gene, $S 28(t)$, on rice chromosome 4. Rice Genetics Newsletters 19:80-82

39. Stein JC, Yu Y, Copetti D, Zwickl DJ, Zhang L, Zhang C, Chougule K, Gao D, Iwata A, Goicoechea JL, Wei S, Wang J, Liao Y, Wang M, Jacquemin J, Becker C, Kudrna D, Zhang J, Londono CEM, Song X, Lee S, Sanchez P, Zuccolo A, Ammiraju JSS, Talag J, Danowitz A, Rivera LF, Gschwend AR, Noutsos C, Wu C, Kao S, Zeng J, Wei F, Zhao Q, Feng Q, El Baidouri M, Carpentier M, Lasserre E, Cooke R, Rosa Farias DD, Da Maia LC, Dos Santos RS, Nyberg KG, McNally KL, Mauleon R, Alexandrov N, Schmutz J, Flowers D, Fan C, Weigel D, Jena KK, Wicker T, Chen M, Han B, Henry R, Hsing YC, Kurata N, de Oliveira AC, Panaud O, Jackson SA, Machado CA, Sanderson MJ, Long M, Ware D, Wing RA (2018) Genomes of 13 domesticated and wild rice relatives highlight genetic conservation, turnover and innovation across the genus Oryza. Nat Genet 50:285-296

40. Sui F, Zhao D, Zhu H, Gong Y, Tang Z, Huang XY, Zhang G, Zhao FJ (2019) Map-based cloning of a new total loss-of-function allele of OsHMA3 causing high cadmium accumulation in rice grain. $J$ Exp Bot 70:2857-2871

41. Teng B, Zeng R, Wang Y, Liu Z, Zhang Z, Zhu H, Ding X, Li W, Zhang G (2012) Detection of allelic variation at the $W x$ locus with single-segment substitution lines in rice (Oryza sativa L.). Mol Breeding 30:583-595

42. Uga Y, Fukuta Y, Cai HW, Iwata H, Ohsawa R, Morishima H, Fujimura T (2003a) Mapping QTLs influencing rice floral morphology using recombinant inbred lines derived from a cross between Oryza sativa L. and Oryza rufipogon Griff.. Theoretical and Applied Genetics 107:218-226

43. Uga Y, Fukuta Y, Ohsawa R, Fujimura T (2003b) Variations of floral traits in Asian cultivated rice (Oryza sativa L.) and its wild relatives (O. rufipogon Griff.). Breed Sci 53:345-352

44. Virmani SS, Athwal DS (1973) Genetic variability in floral characteristics influencing outcrossing in Oryza sativa L. Crop Sci 13:66-67

45. Wang S, Wu K, Yuan Q, Liu X, Liu Z, Lin X, Zeng R, Zhu H, Dong G, Qian Q, Zhang G, Fu X (2012) Control of grain size, shape and quality by OSSPL 16 in rice. Nat Genet 44:950-954

46. Wissuwa M, Wegner J, Ae N, Yano M (2002) Substitution mapping of Pup1: a major QTL increasing phosphorus uptake of rice from a phosphorus-deficient soil. Theor Appl Genet 105:890-897

47. Xi Z, He F, Zeng R, Zhang Z, Ding X, Li W, Zhang G (2006) Development of a wide population of chromosome single-segment substitution lines in the genetic background of an elite cultivar of rice (Oryza sativa L.). Genome 49:476-484

48. Xiong LZ, Liu KD, Dai XK, Xu CG, Zhang Q (1999) Identification of genetic factors controlling domestication-related traits of rice using an $\mathrm{F}_{2}$ population of a cross between Oryza sativa and $O$. 
rufipogon. Theoretical and Applied Genetics 98:243-251

49. Xu S, Zheng Y, Liu Y, Guo X, Tan Y, Qian Q, Shu Q, Huang J (2019) Identification of a major quantitative trait locus and its candidate underlying genetic variation for rice stigma exsertion rate. The Crop Journal 7:350-359

50. Yang T, Zhang S, Zhao J, Liu Q, Huang Z, Mao X, Dong J, Wang X, Zhang G, Liu B (2016) Identification and pyramiding of QTLs for cold tolerance at the bud bursting and the seedling stages by use of single segment substitution lines in rice (Oryza sativa L.). Mol Breeding 36:96

51. Yang W, Guo Z, Huang C, Duan L, Chen G, Jiang N, Fang W, Feng H, Xie W, Lian X, Wang G, Luo Q, Zhang Q, Liu Q, Xiong L (2014) Combining high-throughput phenotyping and genome-wide association studies to reveal natural genetic variation in rice. Nat Commun 5:5087

52. Yue GH, Mei HW, Pan BR, Lou J, Li MS, Luo LJ (2009) Mapping of QTLs affecting stigma exsertion rate of Huhan $1 \mathrm{~B}$ as a CMS maintainer of upland hybrid rice. Acta Agriculturae Zhejiangensis 21:241-245 (Chinese with English abstract)

53. Zhang GQ (2019) The platform of breeding by design based on the SSSL library in rice. Hereditas (Beijing) 41:754-760 (Chinese with English abstract)

54. Zhang GQ, Zeng RZ, Zhang ZM, Ding XH, Li WT, Liu GF, He FH, Tulukdar A, Huang CF, Xi ZY, Qin LJ, Shi JQ, Zhao FM, Feng MJ, Shan ZL, Chen L, Guo XQ, Zhu HT, Lu YG (2004) The construction of a library of single segment substitution lines in rice (Oryza sativa L.). Rice Genetics Newsletters 21:8587

55. Zhang K, Zhang Y, Wu W, Zhan X, Anis GB, Rahman MH, Hong Y, Riaz A, Zhu A, Cao Y, Sun L, Yang Z, Yang Q, Cao L, Cheng S (2018) qSE7 is a major quantitative trait locus (QTL) influencing stigma exsertion rate in rice (Oryza sativa L.). Sci Rep 8:14523

56. Zhang Y, Yang J, Shan Z, Shen C, Qiao W, Zhu X, Xie Q, Zhu H, Zhang Z, Zeng R (2012) Substitution mapping of QTLs for blast resistance with SSSLs in rice (Oryza sativa L.). Euphytica 184:141-150

57. Zhang Y, Zhou J, Yang Y, Li J, Xu P, Deng X, Deng W, Wu Z, Tao D (2015) A novel gene responsible for erect panicle from Oryza glumaepatula. Euphytica 205:739-745

58. Zhao H, Sun L, Xiong T, Wang Z, Liao Y, Zou T, Zheng M, Zhang Z, Pan X, He N, Zhang G, Zhu H, Liu Z, He P, Fu X (2019) Genetic characterization of the chromosome single-segment substitution lines of $O$. glumaepatula and $O$. barthii and identification of QTLs for yield-related traits. Mol Breeding 39:51

59. Zhou H, Li P, Xie W, Hussain S, Li Y, Xia D, Zhao H, Sun S, Chen J, Ye H, Hou J, Zhao D, Gao G, Zhang Q, Wang G, Lian X, Xiao J, Yu S, Li X, He Y (2017a) Genome-wide association analyses reveal the genetic basis of stigma exsertion in rice. Mol Plant 10:634-644

60. Zhou Y, Xie Y, Cai J, Liu C, Zhu H, Jiang R, Zhong Y, Zhang G, Tan B, Liu G, Fu X, Liu Z, Wang S, Zhang G, Zeng R (2017b) Substitution mapping of QTLs controlling seed dormancy using single segment substitution lines derived from multiple cultivated rice donors in seven cropping seasons. Theor Appl Genet 130:1191-1205

61. Zuo J, Li J (2014) Molecular dissection of complex agronomic traits of rice: a team effort by chinese scientists in recent years. National Science Review 1:253-276 
Figures

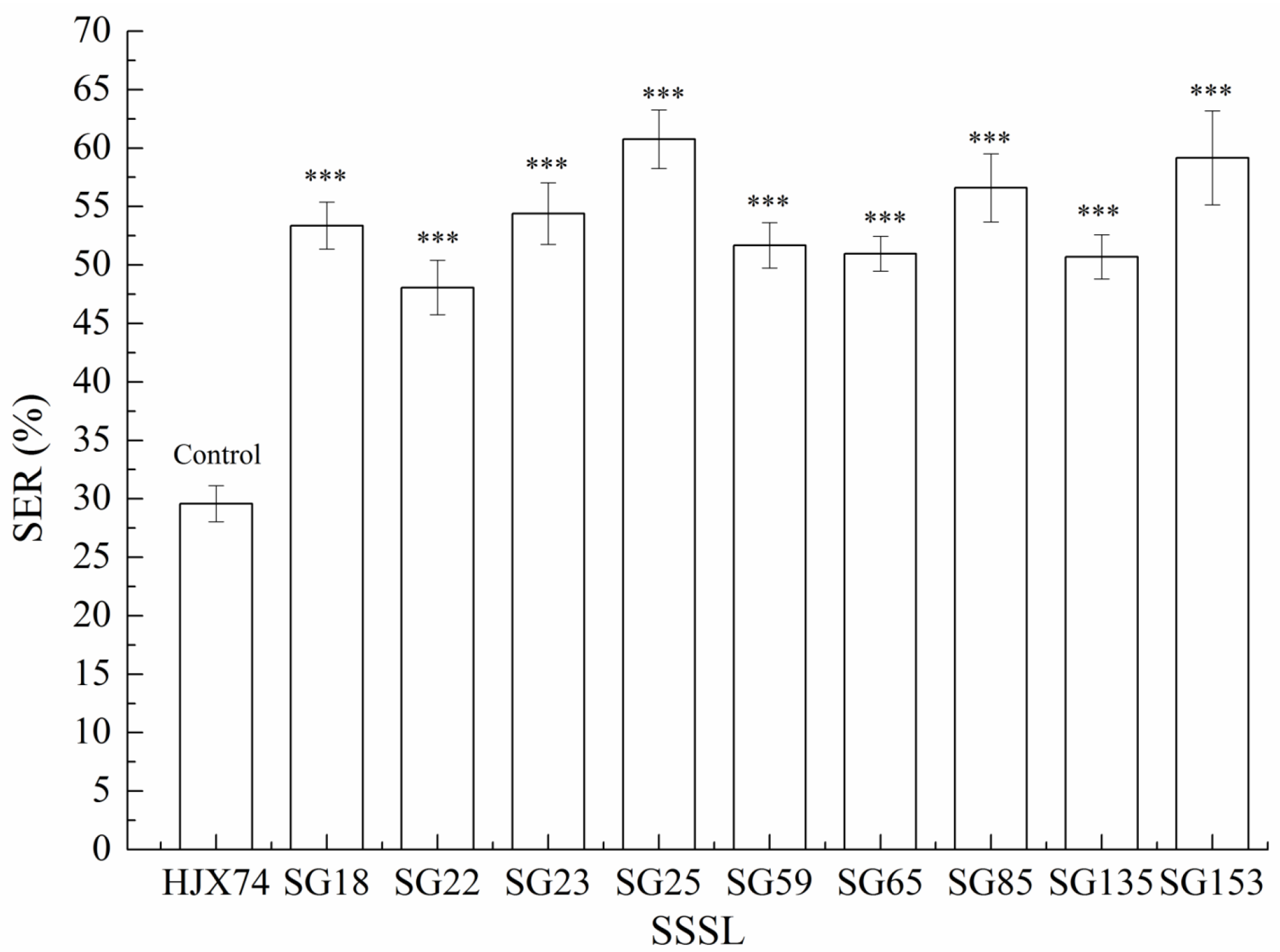

Figure 1

Stigma exsertion rate in the 9 SSSLs and HJX74. SSSL: single-segment substitution line. SER: stigma exsertion rate. Data were presented as mean \pm S.E. in five cropping seasons, one-way ANOVA, LSD, twotailed, ${ }^{\star \star \star} p$-value $\leq 0.001$. The percentages were converted to arcsine values. 


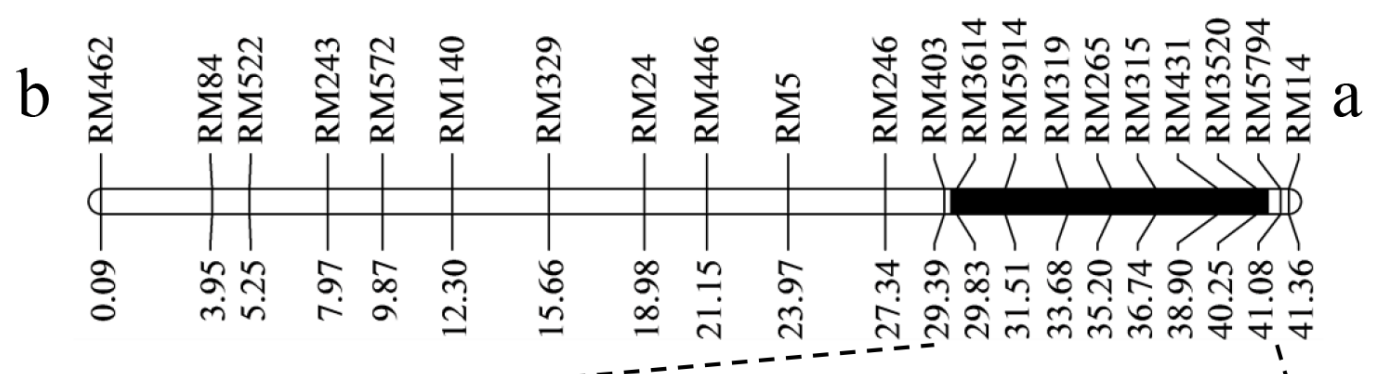

c

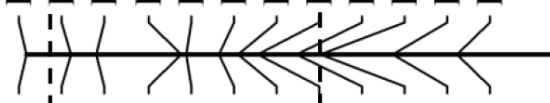

ले

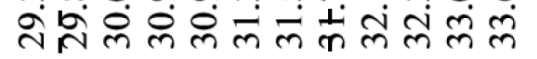

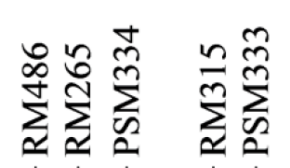

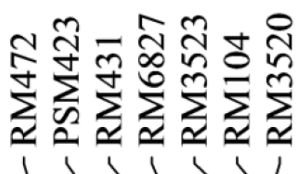

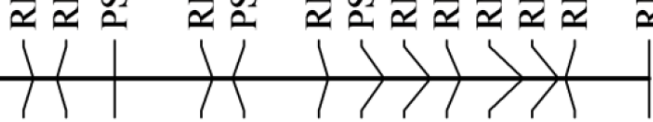

유요

交a

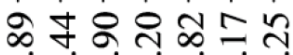

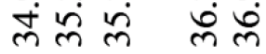

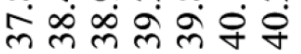
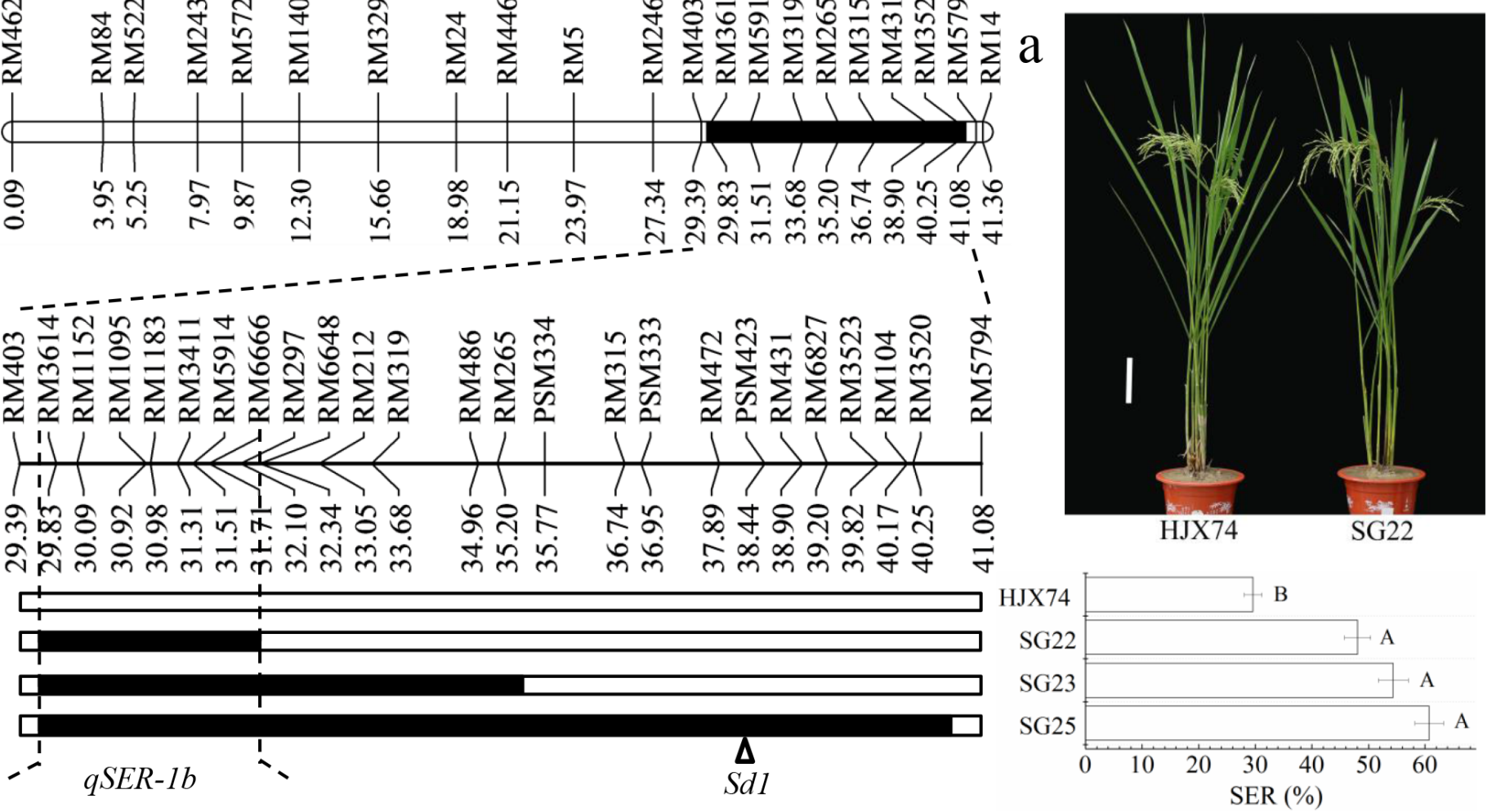

Figure 2

Substitution mapping of qSER-1b. a, Morphology of HJX74 and SG22. Scale bar, $15 \mathrm{~cm}$. b, The region of substituted segments of three SSSLs, SG22, SG23 and SG25, on chromosome 1. c, Substitution mapping of qSER-1b. Stigma exsertion rate (SER) (\%) is a mean \pm S.E. in five cropping seasons. White and black blocks on chromosomes represent genotypes of HJX74 and donor, respectively. Threshold, $a=0.001$, arcsine value, one-way ANOVA, Duncan, two-tailed. The triangle represents the locus of Sd1 (Sasaki et al. 2002). 


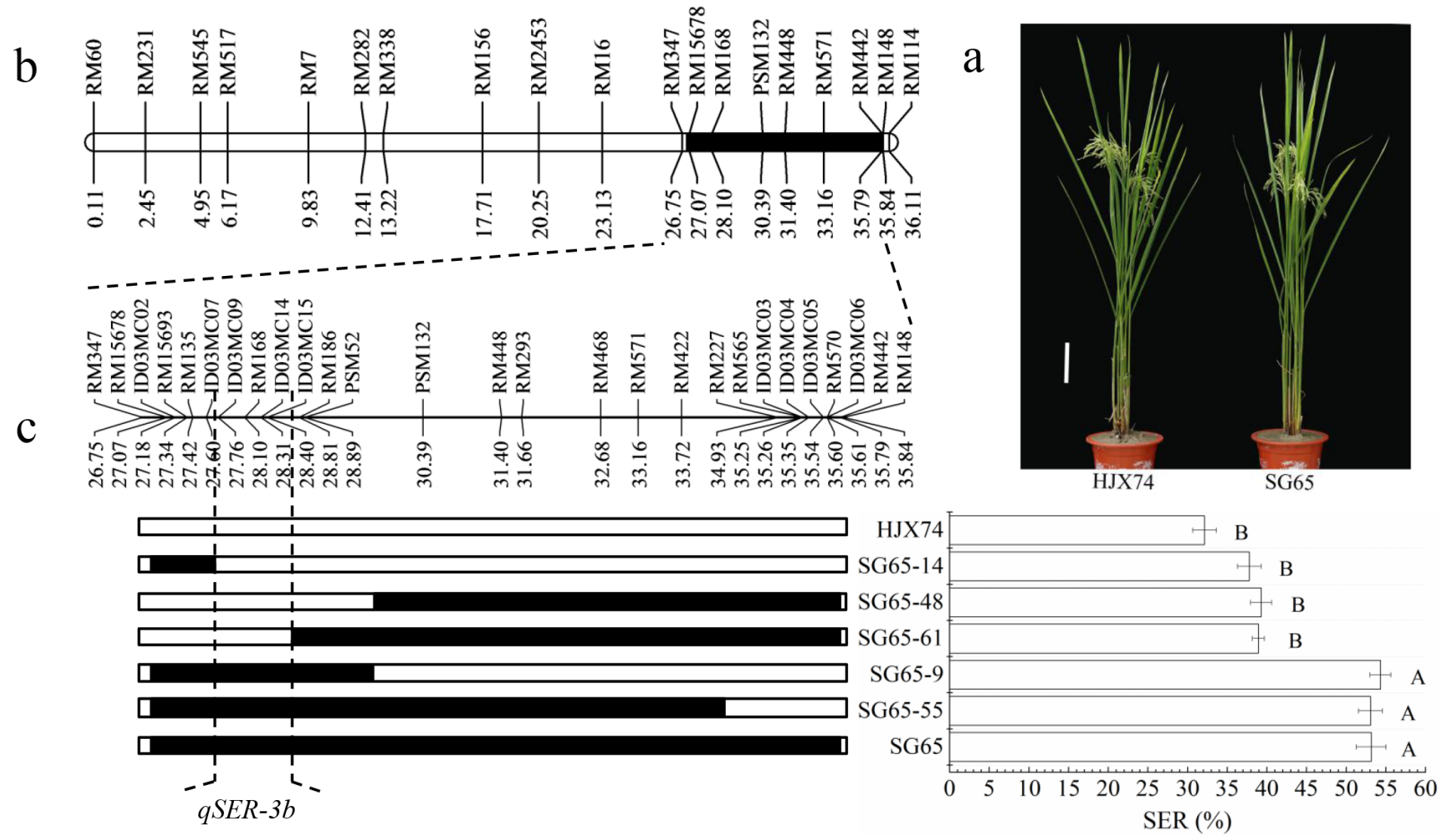

\section{Figure 3}

Secondary substitution mapping of qSER-3b. a, Morphology of HJX74 and SG65. Scale bar, 15 cm. b, The substituted segment of SG65 on chromosome 3. c, Secondary substituted mapping of qSER-3b in the substituted segment of SG65. Stigma exsertion rate (SER) (\%) is a mean \pm S.E.. White and black blocks on chromosomes represent genotypes of HJX74 and donor, respectively. Threshold, $a=0.001$, arcsine value, one-way ANOVA, Duncan, two-tailed. 

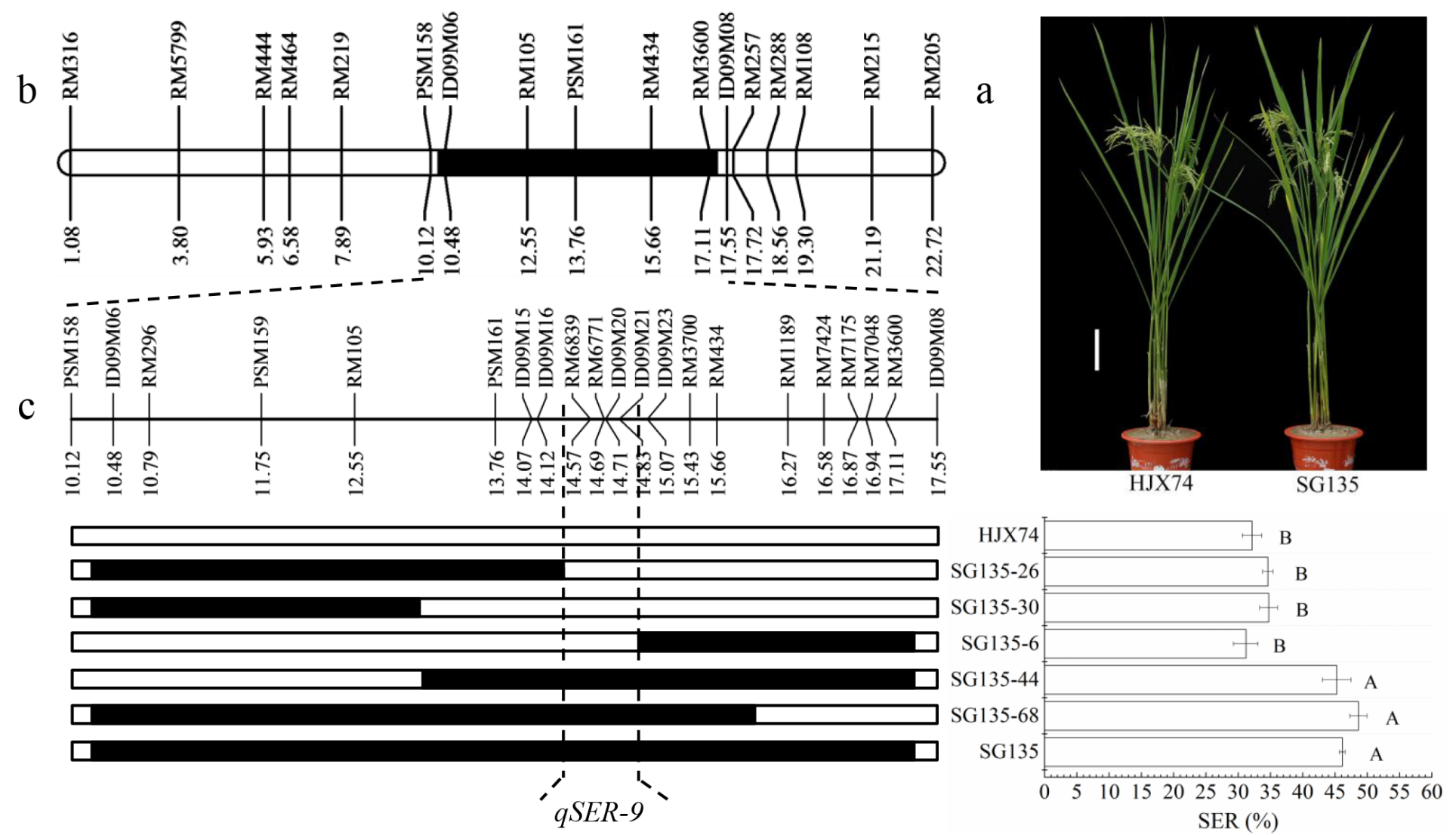

\section{Figure 4}

Secondary substitution mapping of qSER-9. a, Morphology of HJX74 and SG135. Scale bar, $15 \mathrm{~cm}$. b, Substituted segment of SG135 on chromosome 9. c, Secondary substituted mapping of qSER-9 in the substituted segment of SG135. Stigma exsertion rate (SER) (\%) of secondary SSSLs is a mean \pm S.E.. White and black blocks on chromosomes represent genotypes of HJX74 and donor, respectively. Threshold, $a=0.001$, arcsine value, one-way ANOVA, Duncan, two-tailed. 
Mb

Chr.1

Chr.3

Chr.5

Chr.9

Chr.10
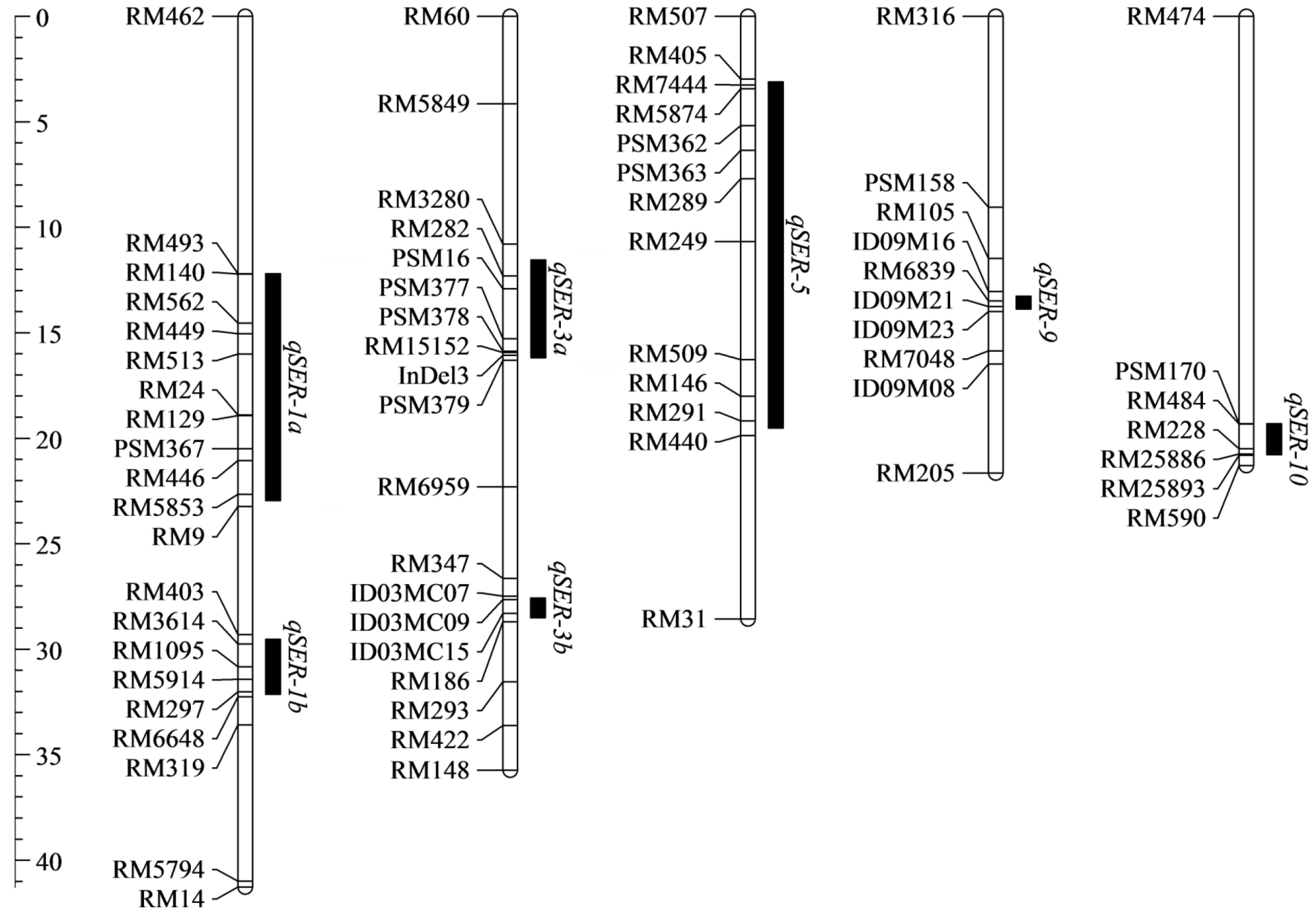

Figure 5

Chromosomal locations of the seven QTLs for SER in the SSSLs. Black bars on the right of each chromosome are the intervals of QTLs with their names on the right. Chr. chromosome, Mb megabase.

\section{Supplementary Files}

This is a list of supplementary files associated with this preprint. Click to download.

- Additionalfile.xlsx 